\title{
Cascaded H-Bridge Multilevel Converter for a High-Power Medium-Voltage Impedance- Admittance Measurement Unit
}

M. Petkovic, N. Hildebrandt, F. D. Freijedo, et al.

This material is posted here with permission of the IEEE. Such permission of the IEEE does not in any way imply IEEE endorsement of any of EPFL's products or services. Internal or personal use of this material is permitted. However, permission to reprint / republish this material for advertising or promotional purposes or for creating new collective works for resale or redistribution must be obtained from the IEEE by writing to pubs-permissions@ieee. org. By choosing to view this document, you agree to all provisions of the copyright laws protecting it. 


\title{
Cascaded H-Bridge Multilevel Converter for a High-Power Medium-Voltage Impedance-Admittance Measurement Unit
}

\author{
Marko Petković, Nicolai Hildebrandt, Francisco D. Freijedo and Dražen Dujić \\ Power Electronics Laboratory - PEL \\ École Polytéchnique Fédérale de Lausanne \\ Station 11, CH-1015 Lausanne, Switzerland \\ marko.petkovic@epfl.ch,nicolai.hildebrandt@epfl.ch,francisco.freijedo@epfl.ch,drazen.dujic@epfl.ch
}

\begin{abstract}
Recent trends in power system design and inclusion of renewable energy sources and smart grids are creating different subsystem interactions that require proper investigation, understanding, describing and estimating global system stability through impedance-admittance measurement and identification. This paper proposes and presents the cascaded $\mathrm{H}$-bridge multilevel inverter topology for perturbation injection converter and impedance-admittance measurement. The methodology of impedance-admittance measurement is explained together with different measurement. Performance and suitability of this topology for impedance-admittance measurement is evaluated through simulations. As a main result of this study, the feasibility of injecting small-signal perturbations in the medium-voltage level system is shown.
\end{abstract}

Index Terms-Cascaded H-Bridge, impedance-admittance measurement, system identification.

\section{INTRODUCTION}

In the wake of strong penetration of renewable energies, smart-grids and traction systems, as an example of interfacing electrical grids of different nature into traditional electrical power systems, power electronics is becoming one of the most important components of modern distributed electrical power system. As an example, Fig. 1 shows the different components and subsystems such as solar fields, wind turbines, storage elements and different ac and dc interface points. Active loads such as power converters are starting to appear next to passive loads. Utilisation of power electronic systems inherently changes the nature of power system by introducing interactions and behaviour that was not present before and is becoming increasingly complex [1], [2]. As a consequence, characterizing power systems and power converter behaviour is of a major interest nowadays. One of most commonly used methods to analyse the behavior and stability of a system is by characterizing small-signal impedance based models and applying the Nyquist criterion to the product between the source output impedance and load input admittance [1]-[3]. This characterization is made possible by the virtue of impedanceadmittance modelling and measurement. For assessing the stability of a whole system, impedance-admittance measurement has to be performed at different interfaces in the system.

978-1-5386-2353-4/18/\$31.00 @ 2018 IEEE
Modern complex power system may be composed of $\mathrm{dc}$ and ac grids of different frequencies and voltage and current levels. Stemming from this, an impedance-admittance measurement devices has to be highly modular and reconfigurable to comply with different constraints at measurement points such as system voltage and current ratings and frequency and the type of connection that can be physically made. In terms of measurements, as wide as possible frequency measurement range is sought for. The precision of measurements on the other side is also of utmost importance. This is due to the fact that the inclusion of power electronic devices, no matter how flexible, versatile and powerful in the sense of enabling the full potential of power systems, also brings some downsides such as harmonics and switching noise polluting the system voltages and currents.

The topic of impedance-admittance measurement for stability analysis of power system has established itself since last decade and there is already a certain amount of work done. In [4] an overview of impedance-admittance measurement techniques based on frequency domain identification techniques for the stability of ac systems is presented. The same work presents different practical implementations for a perturbation injection converter (PIC). Another approach is using the time-domain identification as in [5] via load-stepping. This method is well suitable for identification of parametrical models and its advantage is in the low number of loadstepping responses needed to identify the system. However, the drawback of this method is in the inability to characterize properly the impedances in the high-frequency range. Crosscorrelation methods for extraction of small-signal impedances after injection of pseudo-random binary sequence (PRBS) are presented in [6]. Methods for measuring impedances in $d q$-domain using ac-sweep and wide bandwidth small-signal injection are presented in [7]-[9].

Different measurement devices are already commercially available for low-power low-voltage ranges for the design of power supplies and the technology is well-established [10][12]. However, medium voltage (MV) impedance-admittance measurement technology is still under development at the moment. Equipment such as programmable ac sources and 


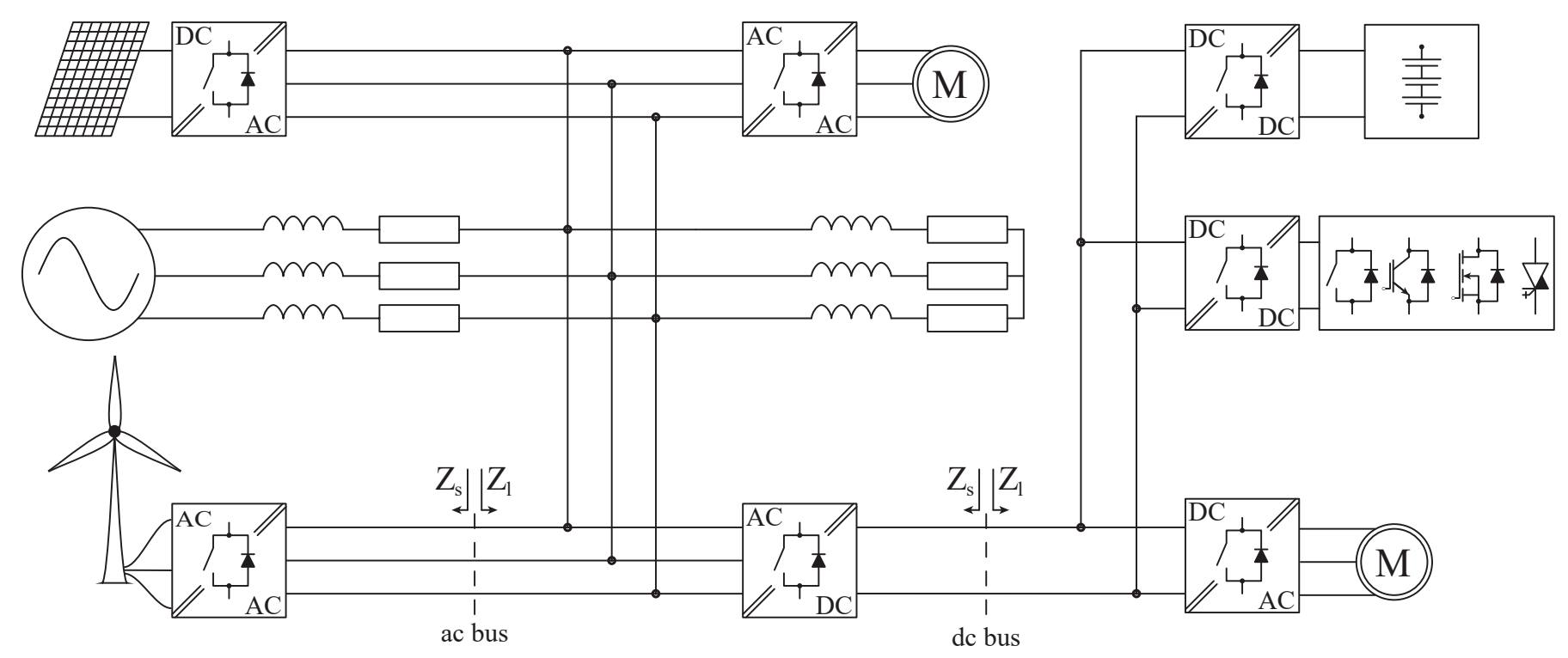

Fig. 1: Modern ac/dc distributed power system in which sources of different nature, ac or dc, and various voltage and current levels are connected through power electronics equipment. At each interface the system can be partitioned into the load and source side where each subsystem has its own characteristic impedance (or admittance) value through which the stability of the system can be assessed.

grid emulators are commercial equipment that could be used for impedance-admittance measurement. They are designed in low voltage (LV) levels of up to $1 \mathrm{kV}$ and power ratings up to $200 \mathrm{kVA}$. These devices are well suited for perturbing systems as they are capable of delivering programmable harmonics or external waveform reference [13]-[16]. In general, this is a good solution when it comes to laboratory experiments in LV levels. However, from a close study in MV applications, we discarded this solution, since using this equipment for MV systems would require a step-up transformer which would in turn cause that the leakage inductances of the transformer would damp the injected perturbation and thus limit the bandwidth of the PIC. This is even more noticeable in higher frequencies. This presents itself as a severe problem during measurements. The perturbation and measured signals should preferably be 10 times bigger than the sensors accuracy which comes as a challenge and a target to achieve. With a proper signal processing strategy this constraint could be relaxed and and a set a more feasible requirement of having a measured signal that is 4 times bigger than the sensor accuracy. These are known as "10:1" and "4:1" rules of thumb in metrology [17].

The previous discussion sets a motivation for our study. A complex research and design problem is established which includes topology identification and choice for PIC, as well as hardware and software design and integration. On the other side, a complex measurement and data acquisition system has to be developed and accuracy and sensitivity to external disturbances of different sensors has to be assessed, considering noisy operational conditions. Once acquired, data has to be processed (online) or post-processed (offline). Then, in order to obtain reliable measurements, different system identification and calibration techniques can be considered. Combination of all of these elements presents itself as a perfect platform for research in medium voltage ac (MVAC) and medium voltage dc (MVDC) impedance-admittance measurements and system identification.

This work presents the selected topology for MVDC and MVAC PIC and investigates its performance potentials. The paper is organised in the following manner: Section II presents perturbation injection and measurement methods giving an overview of how the PIC can be interfaced to system. Section III presents the topology chosen and its different most important parts. Section IV presents the simulations and performance of PIC in the environment of PIC and finally Section V concludes the work and discusses the result

\section{Pertubation injection CONVERTER And MEASUREMENT METHODS}

Different methods of connecting a perturbation devices to a system to be identified have been researched and published. Main methods that are of interest in this work are presented in Fig. 2. In all of these variants a small-signal perturbations, voltage or current, are injected into an unknown system, then current and voltage responses are measured and from these the impedances are calculated and the system impedance (or admittance) is identified. This work considers these three connection methods as it offers a flexibility in terms of type of systems to be identified.

In the case of Fig. 2a the PIC is a main source and an injection device at the same time, a high frequency signal is injected on top of a fundamental one. In this approach there 


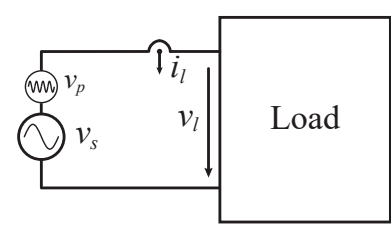

(a)

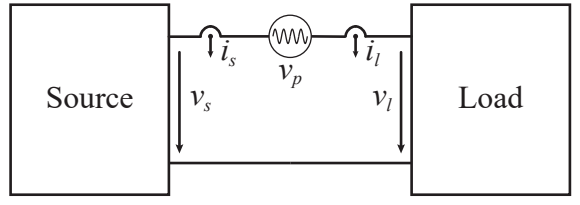

(b)

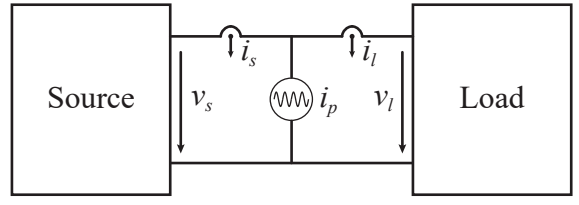

(c)

Fig. 2: Different injections methods of small signals for system identification. a) Pertubation by PIC interfacing a load and perturbing with a small voltage on top of the fundamental wave. b) Perturbation by voltage source connected in series between source and load. c) Pertubation by current source connected in shunt between source and load.

is only one load and is well suited for grid emulators such as those in [13]-[16].

In Fig. $2 b$ the PIC is connected in series between the source and the load subsystems and is used to inject small-signal voltage perturbations in the system. In this case the injected voltage has to be synchronized with the source voltage at the injection point. Advantage of this method is that the power of the small-signal perturbation is low. However, this method presents itself with difficulties when it comes to PIC shortcircuit protection and the PIC has to support rated system current.

Fig. 2c presents a shunt connected device between the source and load subsystems for small-signal current injection. As in the previous way, there has to exist a mean of synchronizing the injected small-signal with the system current. Advantage of this method is that the system current does not flow through the PIC unlike with the previous method, but on the other hand the PIC has to withstand the full line voltage of the system. Current perturbation injection is better suited for identifying smaller source side impedances as often larger load side impedance will force the injected current to flow in the direction of the source, thus leaving little perturbation current for the load side and making the load side measurement and identification more difficult. Another drawback is the fact that it is difficult to inject controlled harmonic current since it closed-loop control is necessary. Connecting a device between two subsystems allows for the device to be an active part of a whole system and at the same time to be a perturbation device. Methods in Fig. 2 are generic ones and present a single-phase connection, but of course they can be extended and applied to three-phase systems which are of interest in this work. There exists a plethora of possible signal types and waveforms that could be used for injection and system perturbation. Most basic form of perturbing a system is using the ac sweep where a single frequency is injected at a time which in the large frequency range of interest makes the measurement considerably slow. Another method is to use wide bandwidth signals such as swept sine in which signal frequency increases (or decreases) over a certain time. Random signals such as PRBS is introduced as white noise approximation, but as true definition. Even though these signals reduce measurement time, over a large frequency range their signal-to-noise ratio (SNR) decreases making the measurement difficult. Another suitable signal is the multisine signal which contains multiple tones at the same time. The SNR of multisine signal is constant over a frequency range but is nevertheless lower compared to a single tone signal used for the ac-sweep. Evidently, a trade-off between the time and ease of measurement has to be made. One of the possible approaches to mitigate the problem of low SNR when using wide bandwidth signals is to use periodogram power spectral density methods [18], [19]. In [20] it is shown that the cross power spectral density (CPSD) estimation based on Welch's periodogram spectral density estimation [21] is successfully used for identifying impedances from noisy measurements.

When it comes to measurements themselves, in the case of Figs. $2 b$ and $2 c$, source and load voltages and currents are measured while in the case of Fig. 2a, source voltage and load current are measured. Voltage and current transducers must have a sufficient accuracy. Not only magnitude but also the phase accuracy has to be high enough as the phase characteristics of sensors will also influence the impedance results [7]. It goes without saying that high enough bandwidth is a must for these transducers. Possible candidates for transducers are capacitive voltage dividers for line voltage measurements and Rogowski and closed loop Hall current sensors for line current measurements.

Concerning voltage and current injection devices, different devices are reported in [22]-[24]. Reference [25] provides results in the MVAC grids and it describes a single phase impedance measurement unit in which three voltage source switching cells are connected in either parallel or series configuration and each of them fed from a dc-link. However, for this work, a highly scalable and modular solution, in terms of voltage and power levels, that can be employed for different applications is sought after. To this end, multilevel topologies appear as a possible candidate for the application. When directly interfacing an MV grid, multilevel converters provide a much reduced short-circuit impedance than solutions based on LV power electronics in combination with stepup transformers. We consider that cascaded H-bridge (CHB) with multipulse transformer better match a realistic / cost effective scenario. On one side these topologies are scalable and versatile, they can be used as a main source and as a perturbation source at the same time. On the other side, in terms of control, multilevel topologies achieve a higher bandwidth (modulation and control) than topologies capable of delivering two level or three level outputs for the same output 
voltage and power rating. Having a higher bandwidth immediately provides higher perturbation frequencies and allows to identify the system up to a higher frequency. Thanks to the multilevel topology, there is no isolating transformer on the output where measurements are performed, thus no reduction on the bandwidth that is now determined by implementation of converter stages. Due to the advantages that this solution possesses, a multilevel CHB inverter topology supplied by a multi-winding transformer is proposed and investigated for PIC application.

\section{CASCADED H-BRIDGE BASED IMPEDANCE-ADMITTANCE MEASUREMENT UNIT}

Different perturbation injection devices have been reported in the literature and all of them suffer from one common downside, the switching noise, as does all the power electronic equipment. When the switching noise is present, together with its side harmonics it becomes much more difficult to identify system response and separate it from the surrounding noise. However, one solution to alleviate this problem, at least to some extent, is to use a CHB multilevel inverter topology. As mentioned in Section II, base for the development of this solution is the multi-winding transformer in Fig. 3. Details about the transformer characteristics and modelling for electric circuit simulations are presented in [26]. The transformer itself shall be interfaced with a multilevel CHB converter (see Fig. 4) and five secondary winding of one single phase are connected to cells which are connected in series at the output. This results in an 11-level CHB inverter topology for the PIC which is the key element of the PIC. Having such a high number of voltage levels in combination with phase-shifted carrier PWM (PSCPWM) gives a less distorted waveform which in return gives less noise pollution and better system response to be identified.

The PIC shall be capable of providing all three connection modes as indicated in Fig. 2. In the series voltage injection mode the cells are connected in parallel, while in shunt current injection mode the cells are connected in series. As the PIC has to be connected in two different ways, the whole system has to be modular and cell connections have to be reconfigurable. The simplest and cheapest way of achieving this reconfigurability is mechanically through the system of bus bars. Most of the reported injection devices are unidirectional and are capable of creating a unidirectional perturbation whereas the topology reported in this work is a four-quadrant one and is capable of sinking current and reversing the voltage polarity which leaves open a question of influence of these methods on impedanceadmittance measurement and system identification. A clear advantage is the fact that the PIC is a grid emulator and a measurement device at the same time. A cell itself is presented in Fig. 5 and it comprises an input LC filter, active front-end (AFE) rectifier and a $\mathrm{H}$-bridge ( $\mathrm{HB}$ ) inverter. Additionally, a voltage limiting unit is also present in the cell. The bandwidth requirements and realization for the $\mathrm{AFE}$ and the $\mathrm{HB}$ are not the same, thus to prevent transient overvoltages in the dc link this protection circuit is used. A series connection of cells

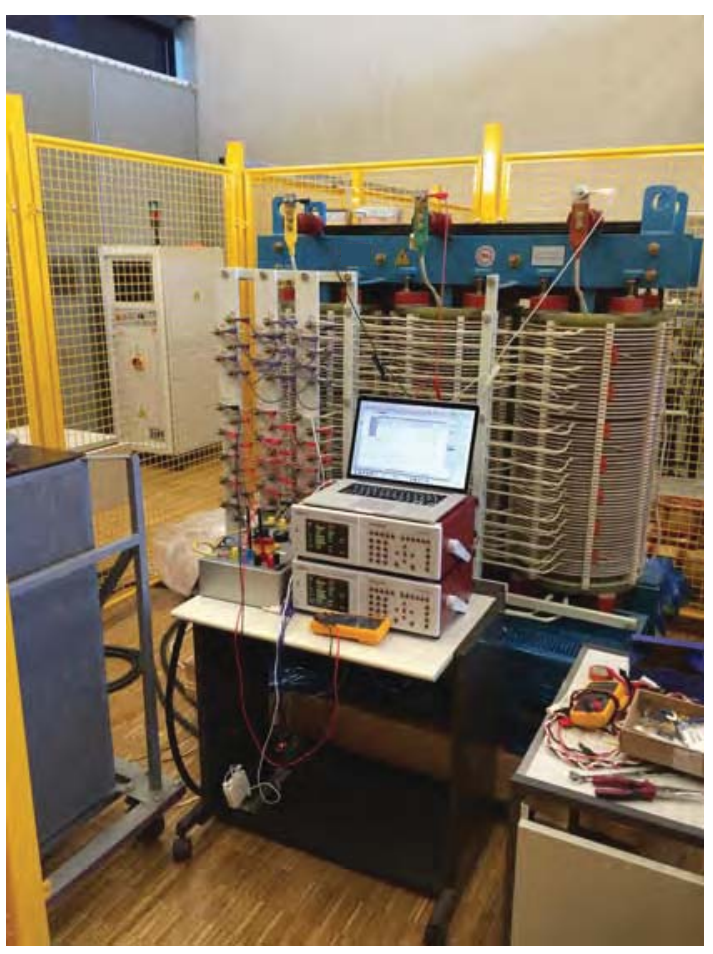

Fig. 3: 1 MVA multi-winding transformer.

TABLE I: Multi-Winding Transformer Parameters

\begin{tabular}{cc}
\hline Parameter & Value \\
\hline \hline Apparent Power Rating & $1 \mathrm{MVA}$ \\
Primary Side Line Voltage & $6 \mathrm{kV}$ \\
Secondary Side Line Voltage & $710 \mathrm{~V}$ \\
Frequency & $50 \mathrm{~Hz}$ \\
Star Primary Windings & 1 \\
Extended Delta Secondary Windings & 15 \\
Phase Shift of the Secondaries & $0^{\circ}, 12^{\circ}, 24^{\circ}, 36^{\circ}, 48^{\circ}$ \\
\hline
\end{tabular}

for shunt current injection and parallel connection of cells for series voltage injection is shown in Fig. 6.

The 1 MVA converter is built of 15 cells which means that each cell has a nominal power of $66.7 \mathrm{kVA}$. Taking into account this requirement, the injection converter shall be designed to support $6 \mathrm{kV}$ line-to-line ac voltages and $100 \mathrm{~A}$ currents with up to $1 \mathrm{MVA}$ output power [27]. The PIC is required to inject only a portion of system voltage or current, namely $5-10 \%$, in the range of $1 \mathrm{~Hz}-10 \mathrm{kHz}$. As a rule of thumb, this means that the apparent switching frequency should be $100 \mathrm{kHz}$. Since the presence of only five secondary windings per phase implies relatively low multiplication factor $(N=5)$ for the apparent switching frequency, it means that the switches inside a HB should be switched at least at $20 \mathrm{kHz}$. Due to the high switching frequency silicon carbide (SiC) devices are considered for the HB stage [27]. Table II summarizes the cell parameters.

\section{A. Active Front-End}

In order to investigate the influence of having a fourquadrant topology for perturbation creation front-end is silicon 


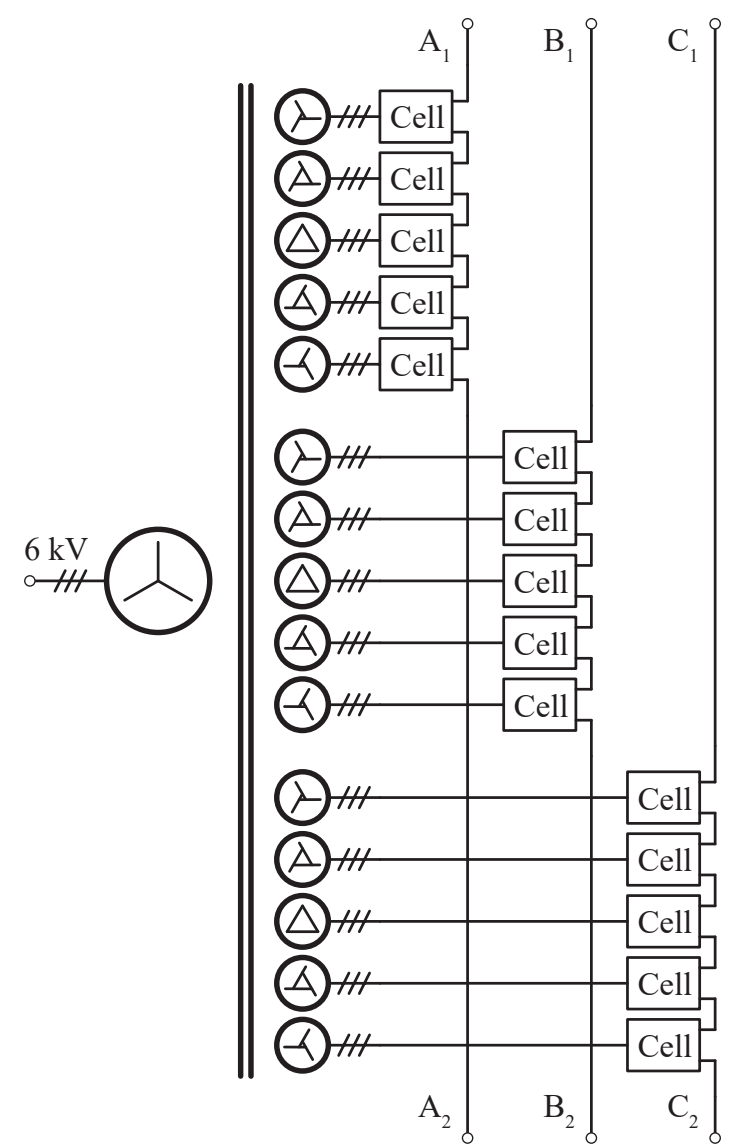

Fig. 4: CHB topology interfaced with a multi-winding transformer.

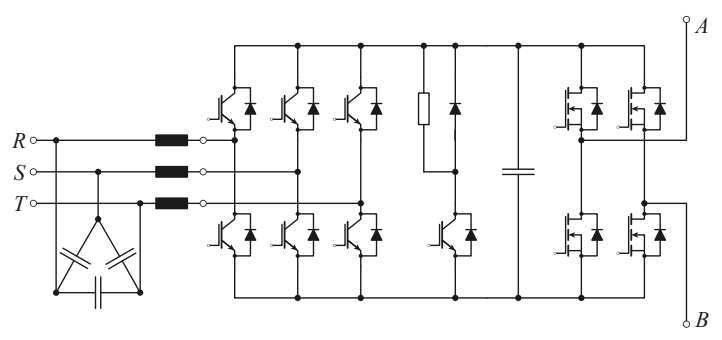

Fig. 5: Regenerative cell with an input LC filter, AFE stage (left) and inverter HB stage (right).

IGBT based AFE. Switching frequency is set at $10 \mathrm{kHz}$. Transformer secondaries provide a multiple of $60^{\circ} / \mathrm{N}$ phase shift. Due to this, the current control bandwidth of AFE is rather low. On the other side, having a high dc-link voltage control bandwidth and lower filtering effort of AFE switching harmonics is sought after. This requirement is filled by increasing the AFE switching frequency. Power modules of the $1.7 \mathrm{kV}$ voltage class are chosen for this application, and for simplicity reasons two-level switching cells are chosen. Reason for choosing this voltage class modules is the nominal dc-link voltage which derives from the trafo secondaries line voltage.

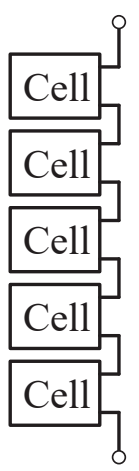

(a)

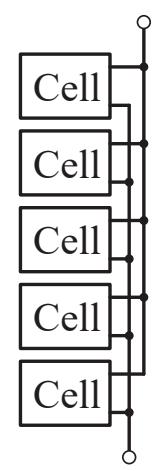

(b)
Fig. 6: A single phase of the CHB topology with a) cells connected in series for shunt current injection and b) cells connected in parallel for series voltage injection.

TABLE II: Cell Parameters

\begin{tabular}{cc}
\hline Parameter & Value \\
\hline \hline Apparent Power Rating & $66.7 \mathrm{kVA}$ \\
AFE Nominal Current & $54 \mathrm{~A}$ \\
Inverter HB Nominal Current & $96 \mathrm{~A}$ \\
Nominal dc-link voltage & $1200 \mathrm{~V}$ \\
Semiconductor blocking voltage & $1700 \mathrm{~V}$ \\
Inverter HB Modulation & PSC-PWM \\
\hline
\end{tabular}

\section{B. DC-link}

The purpose of the dc-link capacitor is dual. One side it serves to maintain the desired dc-link voltage, thus enabling proper dc operation point for the converter, while on the other it serves as a storage for reactive energy during low frequency injection. Unlike the usual design where low frequencies would not be considered, in this case the dc-link design needs to take into account the lowest injection frequency that needs to be achieved while at the same time taking into account the maximum allowable voltage ripple. The dc capacitor can be calculated with respect to the maximum allowed voltage ripple for the lowest injection frequency point [20]. Trying to fulfill all conditions of having highest possible small-signal voltage and current injection and lowest frequency would result in capacitance values far too big for any practical implementation which in turns forces us to make certain trade-offs.

\section{H-Bridge Inverter}

As higher switching frequencies are required at the inverter end, SiC MOSFET devices are chosen for this application. The inverter nominal current is $96 \mathrm{~A}$ so power modules with at least $150 \mathrm{~A}$ current rating are chosen.

\section{Output Waveform Filtering}

Due to the requirement that the PIC has to inject clean highfrequency small-signal voltage and current perturbations up to $10 \mathrm{kHz}$, the inverter end has to comprise a filter to filter pulsewidth modulation (PWM) waveforms. The inverter rectangular shaped voltages can be filtered in two ways, using the $d v / d t$ and sine wave filters. 
The first method filters only high frequency components to reduce the voltage slope of the PWM rectangle outputs. Since the fast switching $\mathrm{SiC}$ MOSFETs are used to synthetize the output waveform their $d v / d t$ can reach high values. In any case of connection to a system seen in Fig. 2 these values have to be limited in order not to provoke a damage to the system being identified. The damped filter resonant frequency $f_{d}$ of this filter is chosen to be higher than both the output frequency $f_{\text {out }}$ and the switching frequency, i.e. $f_{\text {out }} \ll f_{\text {sw }} \ll f_{d}$.

The second approach is used to filter out the switching frequency components to obtain a sinusoidal output waveform. Three factors determine the choice of the sinusoidal filter. The first is the tolerance of the voltage deformation level of the output filter, the second one is the maximum allowable voltage drop on the filter and the third depends on the switching frequency of the inverter switches [28]. The first two factors are directly reflected on the output waveform of the PIC. Third factor reflects on the damped resonant frequency which is required to be larger than the output frequency but smaller than the switching frequency, i.e. $f_{\text {out }} \ll f_{d} \ll f_{\text {sw }}$.

Achieving a damped filter resonant frequency for the $d v / d t$ is relatively easy while achieving the same thing for the sine wave filter possesses the problem in the sense that the maximum output frequency of $10 \mathrm{kHz}$ and the apparent switching frequency of $100 \mathrm{kHz}$ are relatively close and filter needs to be carefully designed to obtain a sufficiently good magnitude and phase characteristics in the passband, proper damping and finally attenuation in the stopband.

\section{Perturbation Injection CONVERTer PERFORMANCE}

Three different configurations presented in Fig. 2 were simulated. PIC is connected to a load of $R_{\text {load }}=36 \Omega$ and $L_{\text {load }}=1 \mathrm{mH}$. Fundamental output line-to-line voltage is $5.4 \mathrm{kV}$ and small-signal voltage is $600 \mathrm{~V}$, i.e. $10 \%$ of maximum line-to-line voltage. In this configuration power output of the PIC is $1 \mathrm{MW}$.

Fig. 7 presents results for the case in which the PIC is the main source and perturbation source, as in Fig. 2 a.

Fig. 8 presents load voltage and currents in the case where the small-signal voltage perturbation is injected in series with a source between the source and the load, as in Fig. 2b. Again, the source voltage is $5.4 \mathrm{kV}$ while the small-signal voltage is $600 \mathrm{~V}$ and the load characteristic is the same.

Fig. 9 presents source voltage and currents in the case where the current perturbation is injected in shunt at a point between the source and the load, as in Fig. 2c. In this case the source voltage is $6 \mathrm{kV}$ and the load characteristic is the same. Current injected by the PIC is $9.6 \mathrm{~A}$. In all three configurations perturbations at $1 \mathrm{~Hz}, 1 \mathrm{kHz}$ and $10 \mathrm{kHz}$ are injected.

\section{CONCLUSION}

This paper focused on the CHB topology employed as a PIC whose endgoal usage is impedance-admittance measurement and system identification. The use of multilevel topology in combination with fast switching devices allows
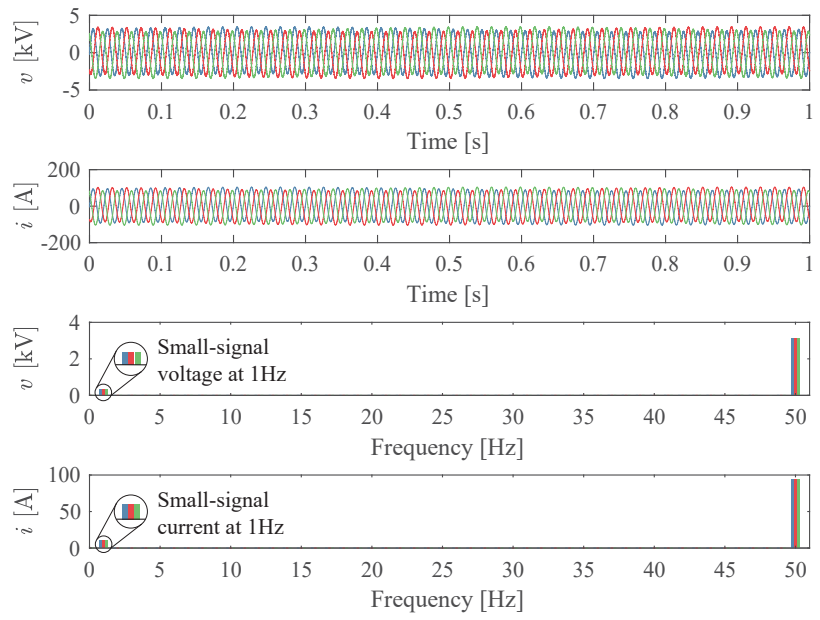

(a) Case of a $1 \mathrm{~Hz}$ injection on top of a $50 \mathrm{~Hz}$ fundamental wave
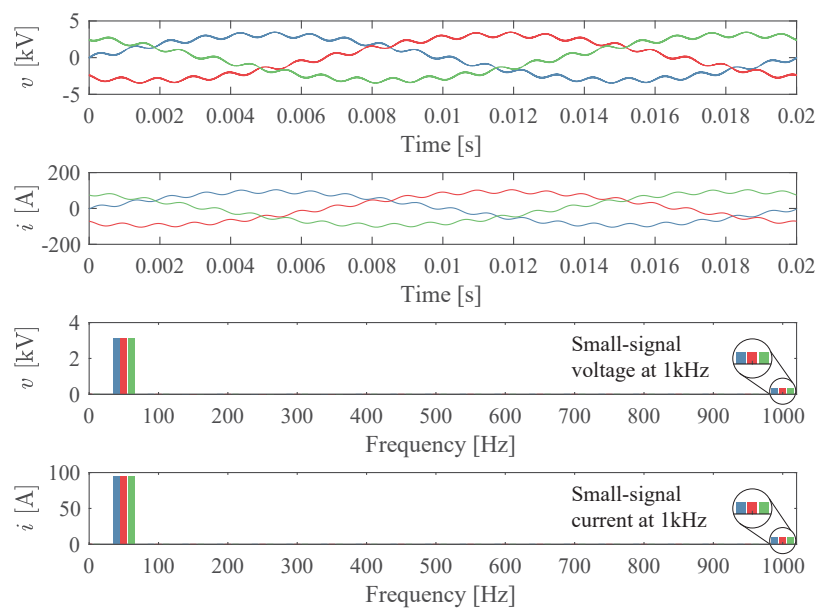

(b) Case of a $1 \mathrm{kHz}$ injection on top of a $50 \mathrm{~Hz}$ fundamental wave
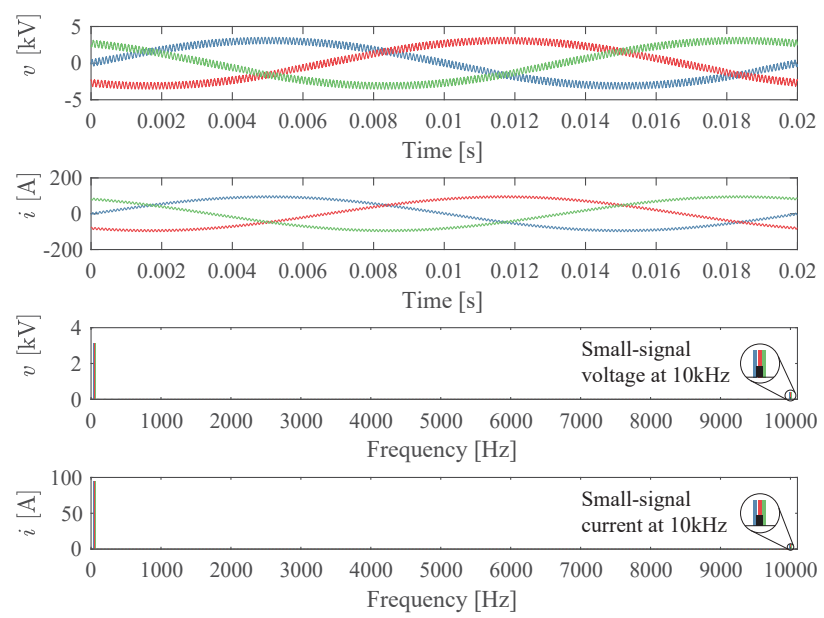

(c) Case of a $10 \mathrm{kHz}$ injection on top of a $50 \mathrm{~Hz}$ fundamental wave

Fig. 7: Simulation waveforms for the configuration in Fig. 2a. $a, b$ and $c$ phase voltages and currents are visible in blue, red and green. 

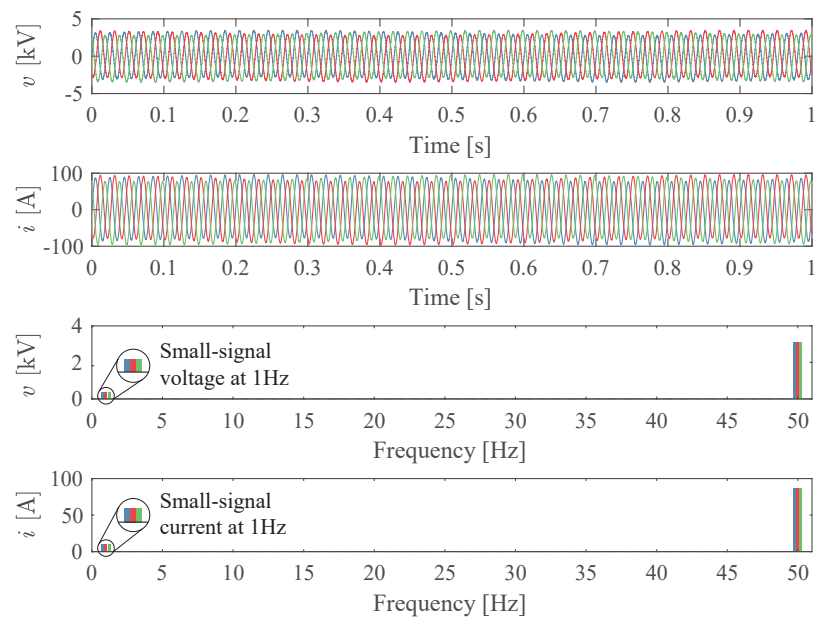

(a) Case of a $1 \mathrm{~Hz}$ series voltage injection into a system with a $50 \mathrm{~Hz}$ fundamental wave
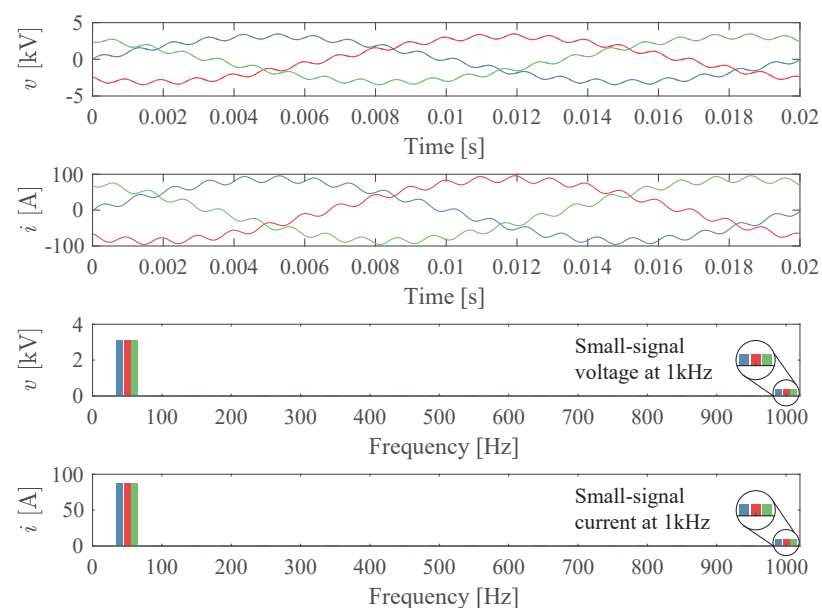

(b) Case of a $1 \mathrm{kHz}$ series voltage injection into a system with a $50 \mathrm{~Hz}$ fundamental wave

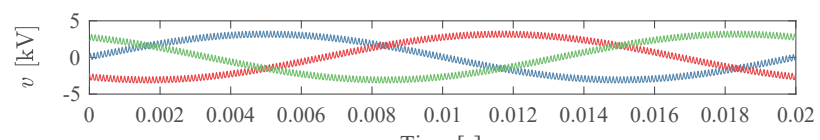
Time $[\mathrm{s}]$
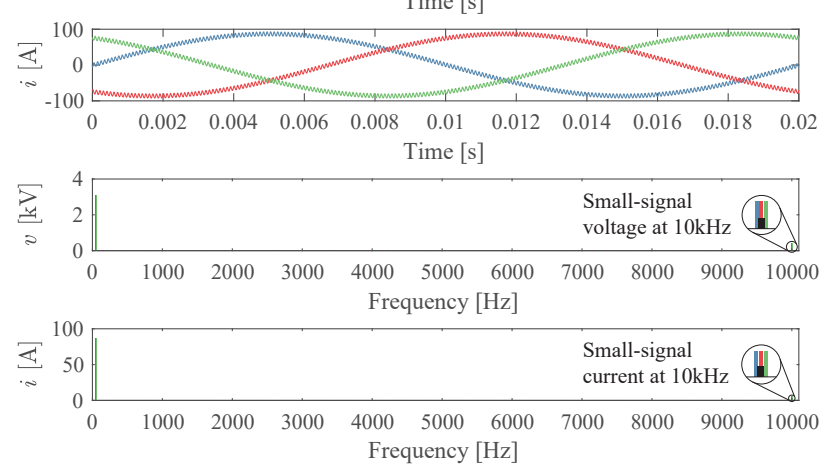

(c) Case of a $10 \mathrm{kHz}$ series voltage injection into a system with a $50 \mathrm{~Hz}$ fundamental wave

Fig. 8: Load side simulation waveforms for the configuration in Fig. 2b. $a, b$ and $c$ phase voltages and currents are visible in blue, red and green.
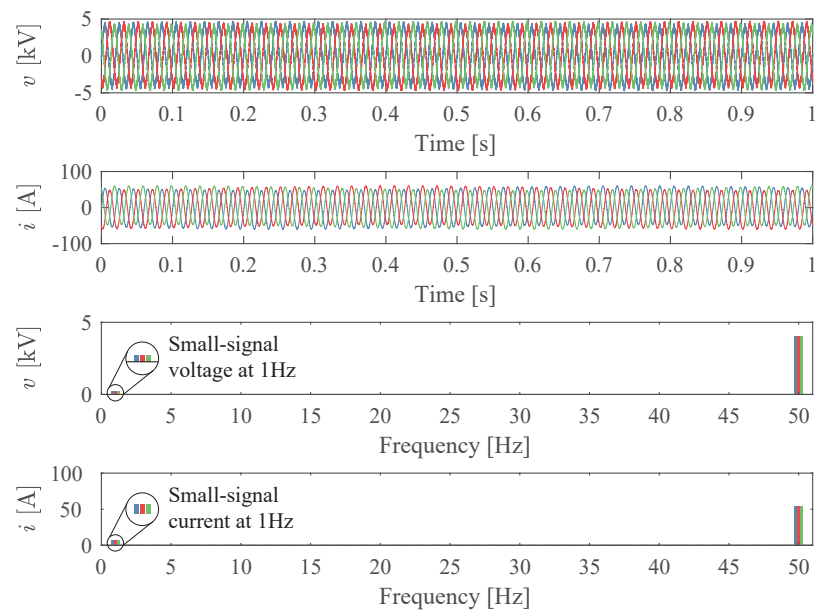

(a) Case of a $1 \mathrm{~Hz}$ shunt current injection into a system with a $50 \mathrm{~Hz}$ fundamental wave
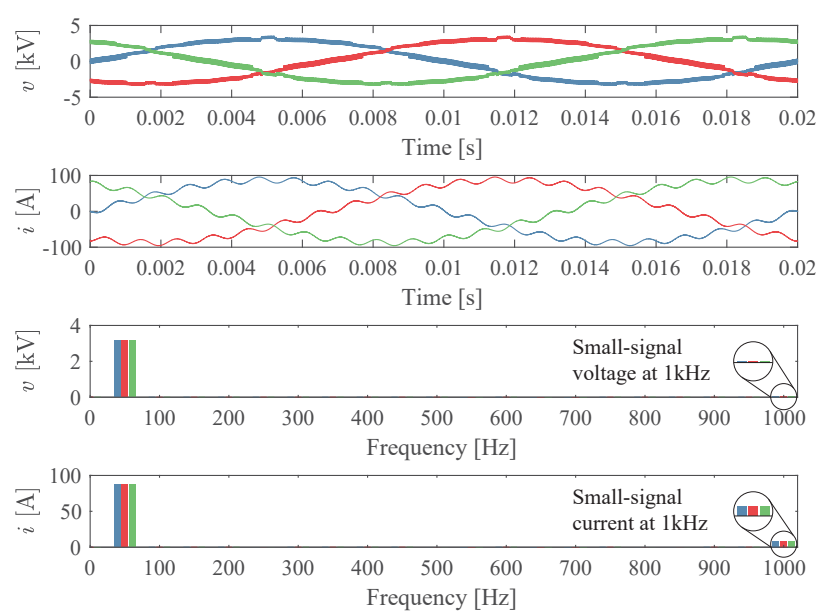

(b) Case of a $1 \mathrm{kHz}$ shunt current injection into a system with a $50 \mathrm{~Hz}$ fundamental wave
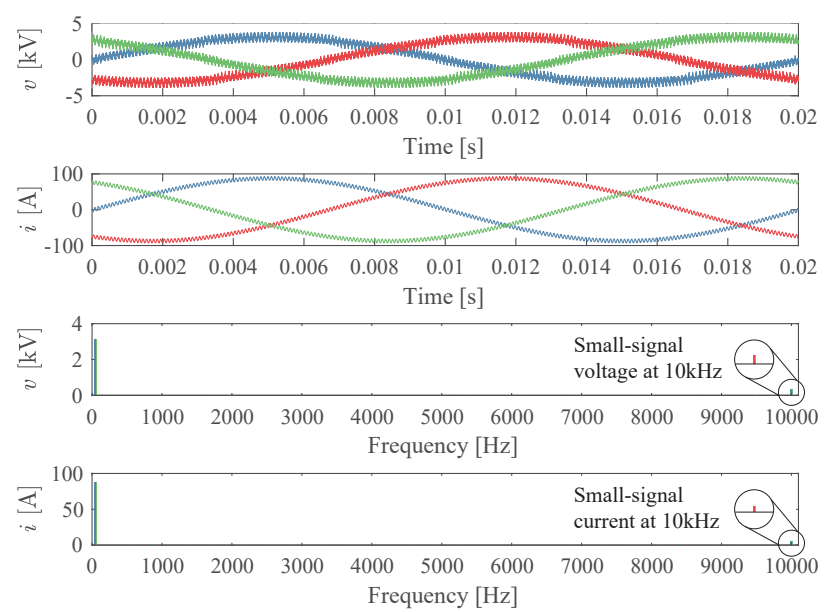

(c) Case of a $10 \mathrm{kHz}$ shunt current injection into a system with a $50 \mathrm{~Hz}$ fundamental wave

Fig. 9: Source side simulation waveforms for the configuration in Fig. 2c. $a, b$ and $c$ phase voltages and currents are visible in blue, red and green. 
for higher apparent switching frequencies and consecutively high frequency output voltage and current which also means a higher bandwidth and better system characterization. Moreover, versatility of the proposed solution provides a capability of having a main source and a perturbation source in the same device, a possibility of connecting the device in three different configurations at various interfaces in the system. Due to the scalability, the proposed topology can be used at different voltage and current levels.

\section{REFERENCES}

[1] X. Wang and F. Blaabjerg, "Harmonic stability in power electronic based power systems: Concept, modeling, and analysis," IEEE Transactions on Smart Grid, 2018.

[2] U. Javaid, F. D. Freijedo, D. Dujic, and W. Van Der Merwe, "Dynamic assessment of source-load interactions in marine mvdc distribution," IEEE Transactions on Industrial Electronics, vol. 64, no. 6, pp. 4372$4381,2017$.

[3] R. D. Middlebrook, "Input filter considerations in design and application of switching regulators," IAS'76, 1976.

[4] Y. A. Familiant, J. Huang, K. A. Corzine, and M. Belkhayat, "New techniques for measuring impedance characteristics of three-phase ac power systems," IEEE Transactions on Power Electronics, vol. 24, no. 7, pp. 1802-1810, 2009.

[5] V. Valdivia, A. Lázaro, A. Barrado, P. Zumel, C. Fernández, and M. Sanz, "Impedance identification procedure of three-phase balanced voltage source inverters based on transient response measurements," IEEE Transactions on Power Electronics, vol. 26, no. 12, pp. 38103816, Dec. 2011.

[6] D. Martin, E. Santi, and A. Barkley, "Wide bandwidth system identification of ac system impedances by applying pertubations to an existing converter," in Energy Conversion Congress and Exposition (ECCE), 2011 IEEE, IEEE, 2011, pp. 2549-2556.

[7] G. Francis, "An algorithm and system for measuring impedance in dq coordinates," PhD thesis, Virginia Tech, 2010.

[8] Z. Shen, "Online measurement of three-phase ac power system impedance in synchronous coordinates," PhD thesis, Virginia Polytechnic Institute and State University, 2013.

[9] G. Francis, R. Burgos, D. Boroyevich, F. Wang, S. Zhiyu, P. Mattavelli, K. Karimi, and S.-w. J. Fu, Algorithm and implementation system for measuring impedance in the $d q$ domain, US Patent 9,140,731, Sep. 2015.

[10] Ridley Engineering. (2016). AP300 Analyzer, [Online]. Available: http: //old.ridleyengineering.com/analyzer.html.

[11] Omicron Lab. (2017). Fast and easy measurements with the Bode 100 [Online]. Available: https://www.omicron-lab.com/bode-100/productdescription.html.

[12] Keysight Technologies. (2018). Impedance Analyzers, [Online]. Available: https://www.keysight.com/en/pc-1000000382\%3Aepsg\%3Apgr/ impedance-analyzers?cc $=$ US\&lc=eng.
[13] Regatron, TopCon TC.ACS - Full 4-quadrant grid simulator, https: //www . regatron . com/en/products - topcon / 18 - english - categories/ products/94-topcon-tc-acs-full-4-quadrant-grid-simulator.

[14] EGSTON, COMPISO P-HIL System, https://egston.com/01-powerelectronics/?lang=en.

[15] Cinergia, GE - Grid Emulator series, http ://www. cinergia . coop/ cinergia-product/ge-grid-emulator-series.

[16] Spitzberger and Spies. (2012). PAS series of 4-Quadrant Amplifi ers, [Online]. Available: www . spitzenberger . de / download . ashx ? weblink=1002.

[17] RFM. (2017). Ten to One or One to Ten Rule, [Online]. Available: https://rfminc.net/resource-center/metrology-tips/ten-to-one-to-ten/.

[18] M. K. Steven et al., "Modern spectral estimation: Theory and application," Signal Processing Series, 1988.

[19] P. Stoica, R. L. Moses, et al., Spectral analysis of signals. Pearson Prentice Hall Upper Saddle River, NJ, 2005, vol. 1, pp. 22-55.

[20] M. D. Jaksic, "Identification of small-signal dq impedances of power electronics converters via single-phase wide-bandwidth injection," $\mathrm{PhD}$ thesis, Virginia Tech, 2015.

[21] P. Welch, "The use of fast fourier transform for the estimation of power spectra: A method based on time averaging over short, modified periodograms," IEEE Transactions on audio and electroacoustics, vol. 15 , no. 2, pp. 70-73, 1967.

[22] Y. L. Familiant, K. A. Corzine, J. Huang, and M. Belkhayat, "Ac impedance measurement techniques," in IEEE International Conference on Electric Machines and Drives, 2005., May 2005, pp. 18501857.

[23] M. Jaksic, Z. Shen, I. Cvetkovic, D. Boroyevich, R. Burgos, and P. Mattavelli, "Wide-bandwidth identification of small-signal dq impedances of ac power systems via single-phase series voltage injection," in 17th European Conference on Power Electronics and Applications (EPE'15 ECCE-Europe), Sep. 2015, pp. 1-10.

[24] Z. Shen, M. Jaksic, P. Mattavelli, D. Boroyevich, J. Verhulst, and M. Belkhayat, "Three-phase ac system impedance measurement unit (imu) using chirp signal injection," in 2013 Twenty-Eighth Аnпиal IEEE Applied Power Electronics Conference and Exposition (APEC), Mar. 2013, pp. 2666-2673.

[25] M. Jakšić, Z. Shen, I. Cvetković, D. Boroyevich, R. Burgos, C. DiMarino, and F. Chen, "Medium-voltage impedance measurement unit for assessing the system stability of electric ships," IEEE Transactions on Energy Conversion, vol. 32, no. 2, pp. 829-841, Jun. 2017.

[26] M. Luo, D. Dujic, and J. Allmeling, "Leakage flux modeling of medium-voltage phase-shift transformers for system-level simulations," IEEE Transactions on Power Electronics, pp. 1-1, 2018, (early access).

[27] N. Hildebrandt, M. Petković, and D. Dujić, "Evaluation of $1.7 \mathrm{kv}$ sic mosfets for a regenerative cascaded h-bridge multilevel converter cell," in 2018 IEEE International Conference on Industrial Technology (ICIT), Feb. 2018, pp. 718-723.

[28] J. Guzinski, H. Abu-Rub, and P. Strankowski, Variable speed AC drives with inverter output filters. John Wiley \& Sons, 2015, pp. 65-93. 